\title{
Preparation of Immobilized Recombinant Tubulin Beta(TuBb) on Chitosan Nanoparticles by Covalent Binding Method
}

\author{
Qingfang Yan ${ }^{\mathrm{a}}$, Yuanyuan Zhang ${ }^{\mathrm{b}}$ and Xiaoying Yin ${ }^{\mathrm{c}^{*}}$ \\ College of Pharmacy, Jiangxi University of Traditional Chinese Medicine, Nanchang 330004, China ${ }^{\text {a }}$ \\ a 869202184@qq.com, ${ }^{\mathrm{b}}$ E-mail:1261115822@qq.com and ${ }^{\mathrm{c}}$ E-mail: ncyxoy@163.com*
}

\begin{abstract}
Objective In order to screen $\mathrm{TuBb}$ inhibitors, this paper describes the preparation of immobilized $\mathrm{TuBb}$ on chitosan nanoparticles. Methods $\mathrm{TuBb}$ was immobilized onto chitosan nanoparticles by covalent binding method. Results The results of the univariate test indicated that the highest immobilized yield can be obtained when the optimal immobilization condition was $1 \mathrm{mg}$ of TuBb, $0.5 \mathrm{~mol} / \mathrm{L}$ of buffer solution with $\mathrm{pH}$ 6.5, immobilization $30 \mathrm{~min}$ and immobilization at $0-4{ }^{\circ} \mathrm{C}$. Conclusions The authors conclude that the immobilized $\mathrm{TuBb}$ maintain the catalysis properties and can be used as the screening of $\mathrm{TuBb}$ inhibitors.

Keywords: $\mathrm{TuBb}$; Chitosan nanoparticles; Immobilization;
\end{abstract}

\section{Introduction}

Microtubule is an important component of the cytoskeleton, which has been demonstrated to be critical in many cellular processes. $\mathrm{TuBb}$ as the basic unit composed of microtubule, has become an important target for anticancer drug research ${ }^{[1]}$. TuBb inhibitors have been considered to be an effective therapeutic approach in the treatment of tumor, such as Taxol and Colchicine ${ }^{[2-3]}$. Traditional Chinese medicines(TCMs) are rich in resources and nowadays, many researchs have been focused on sceening enzyme inhibitors from TCMs ${ }^{[4-}$ 5]. However, the complex composition make it difficult to develop a simple, rapid and reliable method for screening TuBb inhibitors from TCMs.

The immobilization of enzyme on suitable carriers have been proved effective in improve $\mathrm{TuBb}$ stability and reusability, while the catalysis properties are maintained. The immobilized enzyme with good performance can be used as the screening of $\mathrm{TuBb}$ inhibitors $^{[6-8]}$.

There are many enzyme immobilization methods and immobilization methods can be roughly summarized as follows: vector adsorption method, including physical adsorption method, covalent

binding method and ionic binding method; crosslinking; embedding method, including microcapsules type and grid type ${ }^{[9-90]}$.

In this dissertation, $\mathrm{TuBb}$ was immobilized on chitosan nanoparticles by covalent binding method. The advantage of covalent binding method is firm combination between

* Corresponding author: Xiao-Ying Yin, E-mail:ncyxoy@163.com 
$\mathrm{TuBb}$ and chitosan nanaparticles, allowing $\mathrm{TuBb}$ to be reused. And a series of studies were conducted,about the methods for $\mathrm{TuBb}$ immobilization and the investigation of immobilization conditions.

\section{Materials and Instruments}

Recombinant tubulin beta was purchased from Cloud-Clone Co., Ltd.. Bovine serum albumin was purchased from Jiangsu Anhui Biotechnology Co., Ltd.. Coomassie brilliant blue G250 was purchased from Wuhan More Biotechnology Co., Ltd.. Glutaraldehyde solution(25\%) was purchased from Sinopharm Chemical Reagent Co.,Ltd.. Morpholineethanesulfonic acid, Magnesium chloride hexahydrate and Ethylenebis(oxyethylenenitrilo) tetraacetic acid was purchased from Aladdin Industrial Co.,Ltd.. Chitosan nanoparticles were prepared by the laboratory own. Distilled water was prepared with demimeralized water.

CHY-2 Temperature oscillator was purchased from Jintan Fu Instrument Co., Ltd.. Sartorius B125s electronic balance was purchased from Sartorius Co.,Ltd.. HJ-5 Multifunctional stirrer and HH-2 Digital thermostat water bath were purchased from Changzhou Guohua Electric Co.,Ltd.. KQ-500B Ultrasonic cleaning machine was purchased from Kunshan Ultrasonic Instrument Co.,Ltd.. GT-10-1 High-speed desktop centrifuge was purchased from Beijing Era Beili centrifuge Co.,Ltd.. PHS-29A PH Meter was purchased from Shanghai REX Instrument Factory. UV2102 UV-visible spectrophotometer was purchased from Unico,American.

\section{Methods}

\subsection{Immobilization of Recombinant Tubulin Beta (TUBb)}

Add $40 \mu \mathrm{L}$ gutaraldehyde solution(25\%) into the chitosan nanoparticles suspension $(0.5 \mathrm{~g} / \mathrm{mL}, 1 \mathrm{~mL})$ and shake $90 \mathrm{~min}$ at $30^{\circ} \mathrm{C}$. Centrifugate, remove the supernatant and wash the precipitate three times. Then, the precipitate was ultrasonic dispersed with $1 \mathrm{~mL}$ distilled water. Add $1 \mathrm{~mL} 1 \mathrm{mg} / \mathrm{mLl} \mathrm{TuBb}$ solution, which was prepared by ph $6.5,0.5 \mathrm{~mol} / \mathrm{L}$ cold MES buffer, into the suspension above and shake $30 \mathrm{~min}$ at $0-4^{\circ} \mathrm{C}$. Centrifugate, remove the supernatant and wash the precipitate three times. After immobilization, the tubulin solution and flushing were collected and measure the amount of $\mathrm{TuBb}$ which was not immobilized by Coomassie Brilliant Blue. At last, the immobilized TuBb was stored at $4{ }^{\circ} \mathrm{C}$.

\subsection{Determination of TuBb Immobilization Yield}

Measure the amount of the free $\mathrm{TuBb}$ which was not immobilized by Coomassie Brilliant Blue. Immobilization yield was determined and calculated by Eq.1.

Immobilization Yield $(\%)=\left(\mathrm{m}-\mathrm{m}_{\mathrm{r}}-\mathrm{m}_{\mathrm{r}}\right) / \mathrm{m}^{*} 100 \%$ (Eq.1) where $\mathrm{m}$ is the initial amount of $\mathrm{TuBb}, \mathrm{m}_{\mathrm{f}}$ is the amount of TuBb in the flushing solution, $\mathrm{m}_{\mathrm{r}}$ is the residual amount of $\mathrm{TuBb}$ in the $\mathrm{TuBb}$ solution after immobilization. 


\section{Investigation of immobilization conditions}

\subsection{Effects of time on immobilization yield}

Add $1 \mathrm{~mL}$ chitosan nanoparticle $(0.5 \mathrm{~g})$ to the test tube and shake $30 \mathrm{~min}$ at $30^{\circ} \mathrm{C}$. Add $1 \mathrm{~mL} 1 \mathrm{mg} / \mathrm{mL}$ TuBb solution, which was prepared by ph $6.5,0.5 \mathrm{~mol} / \mathrm{L}$ cold MES buffer, into the suspension above and shake $10,15,20,30,40,50 \mathrm{~min}$ at $0-4{ }^{\circ} \mathrm{C}$. Centrifugate, remove the supernatant and wash the precipitate three times. After immobilization, the tubulin solution and flushing were collected and measure the amount of $\mathrm{TuBb}$ which was not immobilized by Coomassie Brilliant Blue to calculate the immobilization yield. TuBb activity is represented by the relative percentages of the highest TuBb activity.

\subsection{Effects of tempreture on immobilization yield}

Add $1 \mathrm{~mL}$ chitosan nanoparticle $(0.5 \mathrm{~g})$ to the test tube and shake $30 \mathrm{~min}$ at $25,30,35,40,45,50,60^{\circ} \mathrm{C}$. Add $1 \mathrm{~mL} 1 \mathrm{mg} / \mathrm{mL}$ TuBb solution, which was prepared by $\mathrm{ph}$ $6.5,0.5 \mathrm{~mol} / \mathrm{L}$ cold MES buffer, into the suspension above and shake $30 \mathrm{~min}$ at $0-4{ }^{\circ} \mathrm{C}$. Centrifugate, remove the supernatant and wash the precipitate three times. After immobilization, the tubulin solution and flushing were collected and measure the amount of $\mathrm{TuBb}$ which was not immobilized by Coomassie Brilliant Blue to calculate the immobilization yield. $\mathrm{TuBb}$ activity is represented by the relative percentages of the highest $\mathrm{TuBb}$ activity.

\subsection{Effects of pH on immobilization yield}

Add $1 \mathrm{ml}$ chitosan nanoparticle $(0.5 \mathrm{~g})$ to the test tube ,adjust $\mathrm{pH}(4.0,4.5,5.0,5 \cdot 5,6.0,6.5,7.0)$ and shake $30 \mathrm{~min}$ at $30^{\circ} \mathrm{C}$. Add $1 \mathrm{~mL} 1 \mathrm{mg} / \mathrm{mL} \mathrm{TuBb}$ solution ,which was prepared by ph $6.5,0.5 \mathrm{~mol} / \mathrm{L}$ cold MES buffer , into the suspension above and shake $30 \mathrm{~min}$ at $0-4^{\circ} \mathrm{C}$. Centrifugate, remove the supernatant and wash the precipitate three times. After immobilization, the tubulin solution and flushing were collected and measure the amount of $\mathrm{TuBb}$ which was not immobilized by Coomassie Brilliant Blue to calculate the immobilization yield. TuBb activity is represented by the relative percentages of the highest $\mathrm{TuBb}$ activity.

\subsection{Effects of the amount of TuBb on immobilization yield}

Add $1 \mathrm{ml}$ chitosan nanoparticle $(0.5 \mathrm{~g})$ to the test tube and shake $30 \mathrm{~min}$ at $30^{\circ} \mathrm{C}$. Add $1 \mathrm{Ml}$ $0.5,0.8,1.0,1.3,1.5 \mathrm{mg} / \mathrm{mL}$ TuBb solution , which was prepared by ph $6.5,0.5 \mathrm{~mol} / 1$ cold MES buffer, into the suspension above and shake $30 \mathrm{~min}$ at $0-4^{\circ} \mathrm{C}$. Centrifugate, remove the supernatant and wash the precipitate three times. After immobilization, the tubulin solution and flushing were collected and measure the amount of $\mathrm{TuBb}$ which was not immobilized by Coomassie Brilliant Blue to calculate the immobilization yield. TuBb activity is represented by the relative percentages of the highest $\mathrm{TuBb}$ activity. 


\section{Results and Discussions}

\subsection{Effects of time on immobilization yield}

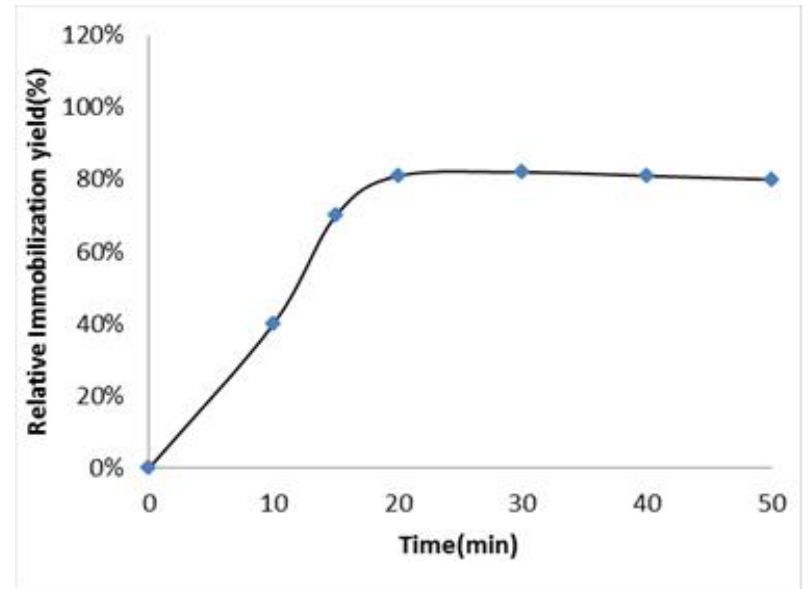

Fig.1 Effects of time on immobilization yield

The Fig. 1 show that when the tempreture is during 0 to $30^{\circ} \mathrm{C}$,relative immobilization yield is rising and when the tempreture is above $30^{\circ} \mathrm{C}$, relative immobilization yield is unchanging. Therefore, we choose $30 \mathrm{~min}$ as the immobilization time.

\subsection{Effects of tempreture on immobilization yield}

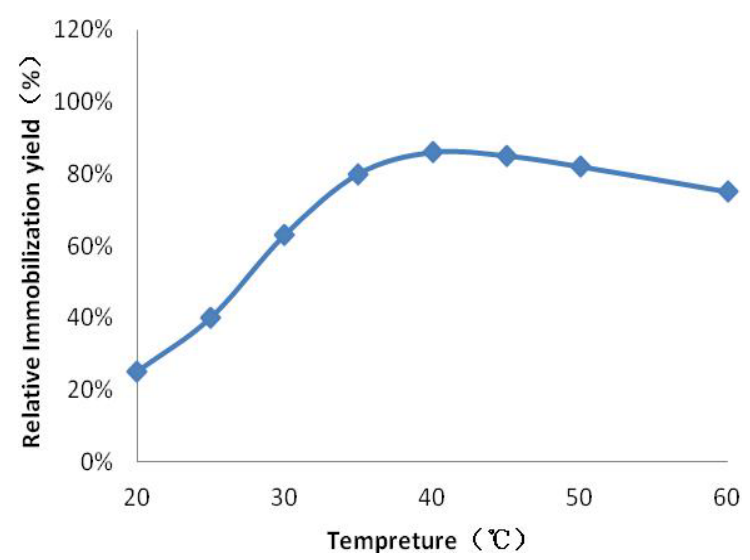

Fig. 2 Effects of tempreture on immobilization yield 
The Fig. 2 show that when the tempreture is during 0 to $40^{\circ} \mathrm{C}$, relative immobilization yield is rising and when the tempreture is above $40^{\circ} \mathrm{C}$, relative immobilization yield is unchanging.In order to retain the activity of $\mathrm{TuBb}$, we choose $0-4^{\circ} \mathrm{C}$ as the immobilization tempreture.

\subsection{Effects of pH on immobilization yield}

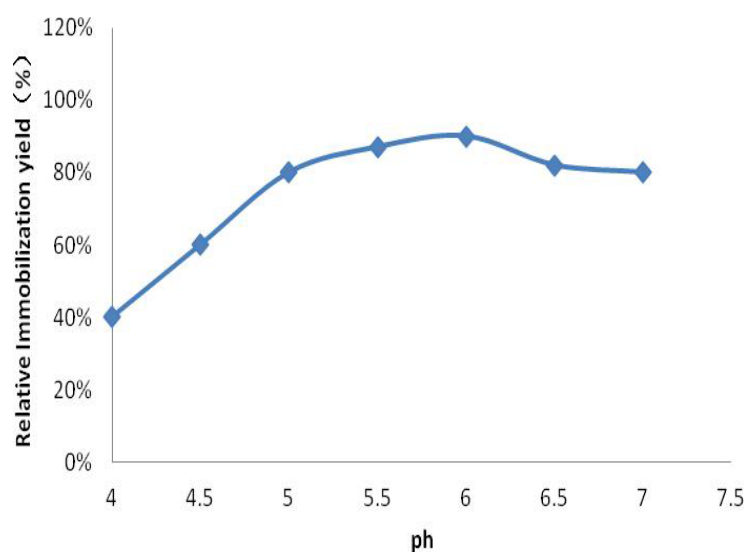

Fig. 3 Effects of $\mathrm{pH}$ on immobilization yield

The Fig. 3 show that when $\mathrm{pH}$ is during 4.0 to 6.0 , relative immobilization yield is rising and when the $\mathrm{pH}$ is above 6.0,relative immobilization yield is reducing. Therefore,we choose $\mathrm{pH} 6.0$ as the $\mathrm{pH}$ of chitosan nanoparticle suspention.

\subsection{Effects of the amount of TuBb on immobilization yield}

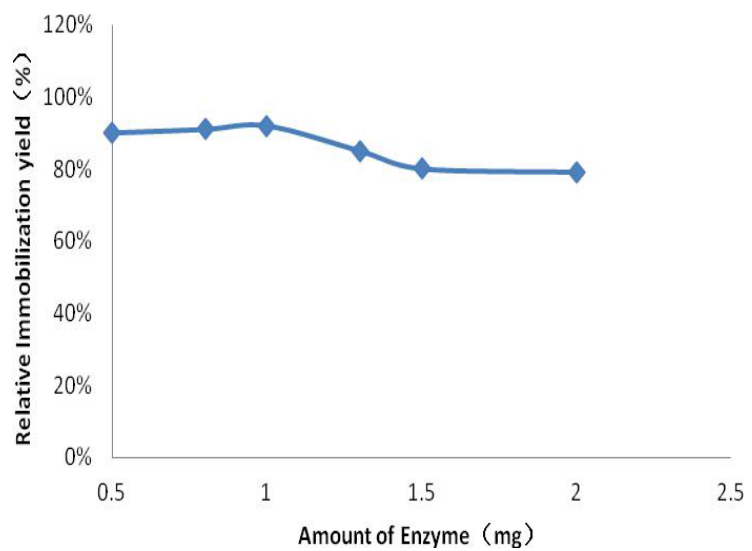

Fig. 4 Effects of the amount of TuBb on immobilization yield 
The Fig. 4 show that when the amount of TuBb is $1.0 \mathrm{mg}$, relative immobilization yield is maxium. Therefore, we choose $1 \mathrm{mg}$ as the immobilization amount of $\mathrm{TuBb}$.

The results of the univariate test indicated that the highest immobilized yield can be obtained when the optimal immobilization condition was $1 \mathrm{mg}$ of $\mathrm{TuBb}, 0.5 \mathrm{~mol} / 1$ of buffer solution with $\mathrm{pH} 6.5$, immobilization $30 \mathrm{~min}$ and immobilization at $0-4^{\circ} \mathrm{C}$.

\section{Conclusions}

In this study, $\mathrm{TuBb}$ was immobilized onto Chitosan nanoparticles and the screening model of $\mathrm{TuBb}$ inhibitors was developped. The screening model exhibited good stability which will prolong the validity of TuBb. Moreover, the model has the potential to be reused, allowing the protein cost to be saved.The method described in this study provides an application example of a rapid, simple and economic approach for the screening of $\mathrm{TuBb}$ inhibitors.

\section{Acknowledge}

This work was financially supported by the National Natural Science Foundation, PR China (Grant No.81260690), the Education Research Project of Science and Technology Foundation,Jiangxi Province,China (Grant No.GJJ13618,GJJ12518) and JiangXi Graduate Student Foundation(Grant No.YC2014-285).

\section{References}

1. Wenna Wang. Design, Synthesis and Biological Evaluation of Benzimidazole deriatives As Novel Tubulin Inhibitors[M].Jilin: Jilin University, 2014: 1-60. In Chinese.

2. Yuma Kudo, Akihiro Abe, et al. Expression of recombinant alpha and beta tubulins from the yew Taxus cuspidate and analysis of the microtubule assembly in the presence of taxol[J].Bioscience,Biotechnology, and Biochemistry,2014,78(11):18871894.

3. Kocha T, Fukuda T, et, al. Hydrophobic chromatography of tubulin on immobilized colchicine columns with various spacers[J].Chemical \& Pharmaceutical Bulletin, 1986,34(9)3791-3802.

4. Dasheng $\mathrm{Lu}$, Jingci $\mathrm{Lv}$,et al. Screening for $\alpha$-glucosidase inhibitors in natural medicines by immobilized enzyme[J].Chinese Journal of New Drugs,2005,14(12):1411-1414.In Chinese.

5. Wenna Wang. Preparation and Application of Immobilized Angiotensin-converting Enzyme on Chitosan[M].Hunan: Hunan Normal University, 2012: 1-117.In Chinese.

6. Simeng Fang, Haina Wang,et al. Immobilized enzyme reactors in HPLC and its application in inhibitor screening:A review[J].Journal of Pharmaceutical Analysis, 2012,2(2): 83-89.

7. Yinglan Nie, Weihong Wang. Immobilized Enzyme Reactor in On-line LC and Its Application in Drug Screening[J]. Chromatographia, 2009(69):S5-S12.

8. Weina Liang,Zhun Hou,et al. Immobilized Enzyme Reactor Chromatography for Online Gelatinase Inhibitors Screening[J].Chromatographia, 2015(78): 763-773.

9. Dambies L, Vincent T, Domard A, et a1. Preparation of chitosan-gel beads by ionotropicmolybdate gelation[J]. Biochemical. Macromolecules, 2001 , 2(4): 1198-1206.

10. Tao G L, Furusaki S . Methods for immobilizing enzymes and some application[J]. Polymer J,1995,27(2):111-116. 